Revista Eletrônica de Ciência Administrativa (RECADM) - ISSN 1677-7387

Faculdade Cenecista de Campo Largo - Coordenação do Curso de Administração

v. 3, n. 2, nov./2004 - http://revistas.facecla.com.br/index.php/recadm/

\title{
EL APRENDIZAJE ORGANIZATIVO: HACIA UN MODELO CONCEPTUAL DE TIPO COGNOSCITIVO
}

\author{
Prof. Dr. Pedro Eugenio López Salazar \\ Profesor Titular de Escuela Universitaria \\ Universidad de Extremadura
}

Facultad de Ciencias Económicas y Empresariales

Departamento de Economía Aplicada y Organización de Empresas

Campus Universitario

Avda. de Elvas s/n

E-06071 Badajoz, España

\section{Prof. José Ángel López Sánchez * \\ Profesor Asociado de Universidad \\ Universidad de Extremadura}

Facultad de Ciencias Económicas y Empresariales

Departamento de Economía Aplicada y Organización de Empresas

Campus Universitario

Avda. de Elvas s/n

E-06071 Badajoz, España

Teléfono: 0034924289300 Ext. 9174

Fax: 0034924272509

E-mail: jangel@unex.es

* Enviar correspondencia a este autor. 
Revista Eletrônica de Ciência Administrativa (RECADM) - ISSN 1677-7387

Faculdade Cenecista de Campo Largo - Coordenação do Curso de Administração

v. 3, n. 2, nov./2004 - http://revistas.facecla.com.br/index.php/recadm/

\title{
EL APRENDIZAJE ORGANIZATIVO: HACIA UN MODELO CONCEPTUAL DE TIPO COGNOSCITIVO
}

\author{
Pedro Eugenio López Salazar, y José Ángel López Sánchez
}

Universidad de Extremadura

\section{RESUMEN}

En este trabajo el objetivo que se persigue es la construcción de un modelo conceptual de aprendizaje organizativo. La motivación principal ha sido la necesidad de entender el papel que desempeñan los modelos mentales en el aprendizaje que se produce, tanto en el ámbito individual como organizativo, ya que la mayoría de marcos teóricos existentes en la literatura especializada que han tratado de explicar este fenómeno lo han ignorado. El modelo propuesto en este sentido mejora la comprensión existente de los procesos de aprendizaje desde un enfoque cognoscitivo: en primer lugar, ofreciendo una visión integrada sobre las conexiones existentes entre el aprendizaje individual y organizativo. En segundo lugar, identificando una serie de impulsores a inhibidores en el proceso de transferencia de aprendizaje.

PALABRAS CLAVE: Aprendizaje Organizativo, Modelo Conceptual, Modelo Mental

\section{ABSTRACT}

The objective of this research is the construction of a conceptual model of organisational learning. Given that the majority of the existing theoretical frameworks in the specialised literature, that have tried to explain the phenomenon of organisational learning, have ignored the influence of mental models, the principal motivation of this investigation has been the need to understand how learning occurs as a result of such mental models, both in the individual and in the organisation. From this point of view, the proposed model improves the current understanding of the learning processes: (1) offering an integrated vision of the existing connections between individual and organisational learning; and (2) identifying a series of factors that encourage or inhibit the process of knowledge transfer.

KEYWORDS: Organisational Learning, Conceptual Model, Mental Models 


\section{Revista Eletrônica de Ciência Administrativa (RECADM) - ISSN 1677-7387 \\ Faculdade Cenecista de Campo Largo - Coordenação do Curso de Administração \\ v. 3, n. 2, nov./2004 - http://revistas.facecla.com.br/index.php/recadm/}

\section{INTRODUCCIÓN}

La relevancia del aprendizaje organizativo está determinada por el advenimiento de la Sociedad del Conocimiento, caracterizada por (Instituto Universitario Euroforum Escorial, 1998, p. 13): (1) la importancia creciente del conocimiento en la actividad económica; (2) el incremento en la velocidad de los cambios; (3) una demanda cada vez más compleja; (4) la revolución tecnológica de las comunicaciones; (5) la globalización de la economía; y (6) unos problemas empresariales más sofisticados.

Estos factores se manifiestan a nivel organizacional de manera más precisa en aspectos tales como: (1) importancia cambiante de los factores de producción; (2) ritmo de cambio acelerado; (3) conocimiento como fuente de ventaja competitiva; (4) consumidores más exigentes; (5) insatisfacción con el paradigma existente en gestión; y (6) aumento en la intensidad de la competencia (Harvey y Denton, 1998, p.210).

Tal nivel de complejidad lleva a un incremento de la incertidumbre, haciendo necesario tanto un mayor nivel de flexibilidad como la creación de nuevas oportunidades de mercado. En este contexto el estudio del aprendizaje organizativo adquiere su relevancia por las siguientes razones:

\$ Busca dar respuesta a los retos que surgen de un entorno empresarial que está en continuo estado de cambio.

\$ Puede ayudar a que las empresas hagan frente a sus dificultades de supervivencia en el largo plazo.

\$ Su análisis puede arrojar luz acerca de uno de los desafíos más importantes en gestión, que no es otro que comprender cuál es el proceso por el que las empresas pueden ser más competitivas.

Al hilo de lo apuntado, cabe destacar que si bien existen diferentes perspectivas teóricas que abordan el estudio del conocimiento como fuente de ventaja competitiva, el interés en este caso se centra en la perspectiva basada en el aprendizaje organizativo. Las otras dos corrientes de investigación son: (1) la perspectiva basada en la gestión del conocimiento; y (2) la perspectiva basada en el capital intelectual. En este estudio el objetivo que se persigue es la construcción de un modelo conceptual de aprendizaje organizativo. La motivación principal ha sido la necesidad de entender el papel que desempeñan los modelos mentales en el aprendizaje que se produce, tanto en el ámbito individual como organizativo, ya que la mayoría de marcos teóricos existentes en la literatura especializada que han tratado de explicar este fenómeno lo han ignorado ${ }^{1}$. Este trabajo se organiza como sigue: en primer lugar, se establecen las premisas del modelo conceptual; en segundo lugar, se analiza el aprendizaje en el ámbito individual; en tercer lugar, se explica el proceso de

\footnotetext{
${ }^{1}$ Aunque no pretende ser una enumeración exhaustiva, algunos de los autores que han ejercido un mayor impacto en investigaciones posteriores, y que han desarrollado un marco teórico con el que se pretenden explicar los procesos de aprendizaje organizativo son: Cangelosi y Dill (1965); Argyris (1977); Shrivastava (1983); Daft y Weick (1984); Fiol y Lyles (1985); Levitt y March (1988); Stata (1989); Senge (1990); Huber (1991); Garvin (1993); Nonaka (1994); y Crossan, Lane y White (1999). Además, se puede agregar el trabajo de Kim (1993), que si bien no ha tenido un impacto tan significatvo en el ámbito del aprendizaje organizativo como los estudios precedentes, es el único que analiza los modelos mentales, aunque no con el nivel de profundidad con que se aborda en la presente investigación.
} 


\section{Revista Eletrônica de Ciência Administrativa (RECADM) - ISSN 1677-7387 \\ Faculdade Cenecista de Campo Largo - Coordenação do Curso de Administração \\ v. 3, n. 2, nov./2004 - http://revistas.facecla.com.br/index.php/recadm/}

transferencia de aprendizaje; por último, se hace una caracterización del mismo examinando Mighty Motors Inc.

\section{LAS PREMISAS DEL MODELO CONCEPTUAL}

La complejidad del logro del objetivo propuesto se fundamenta en el hecho de que: por una parte, se incorporan procesos organizativos y variables que no se habían contemplado con anterioridad en otras perspectivas. Por otra, se integran multitud de ideas dispersas y fragmentadas, a la vez que hechos demostrados empíricamente. Asimismo, la dificultad se ve incrementada por el escaso conocimiento que se tiene de cómo los modelos mentales pueden favorecer el aprendizaje organizativo, y viceversa ${ }^{2}$.

Para el desarrollo del modelo se ha seguido el enfoque metodológico de Crossan, Lane y White (1999), teniendo en cuenta los siguientes elementos: (1) determinación del fenómeno de interés, que en este caso han sido los modelos mentales en el contexto del aprendizaje organizativo; (2) examen de los supuestos subyacentes al modelo conceptual que se propone; y (3) análisis de la relación existente entre los elementos que integran el modelo (ver Figura 1). Las seis premisas o supuestos del modelo son:

Premisa 1: El aprendizaje organizativo es un recurso.

Premisa 2: Los individuos tanto de forma individual como de forma colectiva se sirven de modelos mentales para interpretar la realidad que les rodea.

Premisa 3: La transferencia de aprendizaje entre el ámbito individual y el ámbito organizativo se produce a través del proceso de interacción de los modelos mentales individuales y los modelos mentales compartidos.

Premisa 4: La transferencia de aprendizaje, entre el ámbito individual y el ámbito organizativo, puede estar influenciada por: mapas cognitivos, diálogo, inercia cognitiva, y lógica dominante.

Premisa 5: Los modelos mentales afectan a la acción, y viceversa.

Premisa 6: El aprendizaje organizativo implica la tensión existente entre la exploración y la explotación.

Como se indica en la Premisa 1, el aprendizaje organizativo es un recurso. El marco teórico general en el que se ubica el modelo conceptual que aquí se propone es el de la teoría de la ventaja de los recursos de Hunt y Morgan $^{3}(1995,1996,1997)$. En el análisis que se efectuó de esta teoría en López y López (2001a, 2001b) se argumentó que el aprendizaje organizativo era un recurso. De acuerdo con estos planteamientos, el aprendizaje organizativo permite a la empresa producir eficientemente y/o

\footnotetext{
${ }^{2}$ Daft y Weick (1984: 284-285) sostienen la dificultad inherente a la construcción de un modelo conceptual que trate de recoger la complejidad de una empresa. Así, estos autores hacen referencia a Pondy y Mitroff (1979) para recordar que, en una escala del 1 al 9 de complejidad, la empresas estarían situadas en el nivel 8.

${ }^{3}$ En Hunt (2000) se examinan once tradiciones de investigación diferentes en las que se sustenta la teoría de la ventaja de los recursos. Así, se reconoce que si bien esta teoría tiene afinidades con las tradiciones precedentes, no se concibe como un "agregado" de las teorías que las integran. De este modo, Hunt afirma que la teoría de la ventaja de los recursos sólo se sirve de aquellas cuestiones de cada tradición, que encajan en la lógica interna de la misma. Por otra parte, Priem y Butler (2001)
} 


\section{Revista Eletrônica de Ciência Administrativa (RECADM) - ISSN 1677-7387 \\ Faculdade Cenecista de Campo Largo - Coordenação do Curso de Administração \\ v. 3, n. 2, nov./2004 - http://revistas.facecla.com.br/index.php/recadm/}

eficazmente una oferta de mercado que tiene valor para uno o varios segmentos de mercado. Esto le posibilita alcanzar posiciones de ventaja competitiva, lo que a su vez puede conducirle a obtener un rendimiento financiero superior. Además, este recurso se caracteriza por ser idiosincrásico para cada empresa, y tener una movilidad imperfecta, lo cual le convierte en una ventaja competitiva difícil de imitar por parte de los competidores, y que puede ser perdurable en el largo plazo.

En la Premisa 2 se señala que los individuos se sirven de modelos mentales, tanto de forma individual como de forma colectiva, para interpretar la realidad que les rodea. En este sentido, los sujetos tienen que hacer frente $a$ un entorno de gran complejidad, dinamismo e incertidumbre. A esto se le une la multitud de estímulos que reciben de la realidad. Los modelos mentales ayudan al individuo a procesar esta gran cantidad de información y a poner en orden un todo fragmentado (Picken y Dess, 1998; Senge, 1998). Esto convierte a los modelos mentales individuales en una representación de la realidad a la que se enfrenta la persona; dicha representación ayudará en su interpretación del entorno. En lo que concierne a la organización, también utiliza modelos mentales para guiar su acción, en este caso a partir de los modelos mentales compartidos -organizativos- que surgen del proceso de revelación e interacción con los modelos mentales individuales (Kim, 1993).

FIGURA 1. MODELO CONCEPTUAL DE APRENDIZAJE ORGANIZATIVO.

indican el poder explicativo de la teoría de la ventaja de los recursos por dos razones: en primer lugar, por su rigurosa formalización y, en segundo lugar, por el enfoque integrador que se sigue en su construcción. 
Revista Eletrônica de Ciência Administrativa (RECADM) - ISSN 1677-7387

Faculdade Cenecista de Campo Largo - Coordenação do Curso de Administração

v. 3, n. 2, nov./2004 - http://revistas.facecla.com.br/index.php/recadm/

Fuente: Elaboración propia.

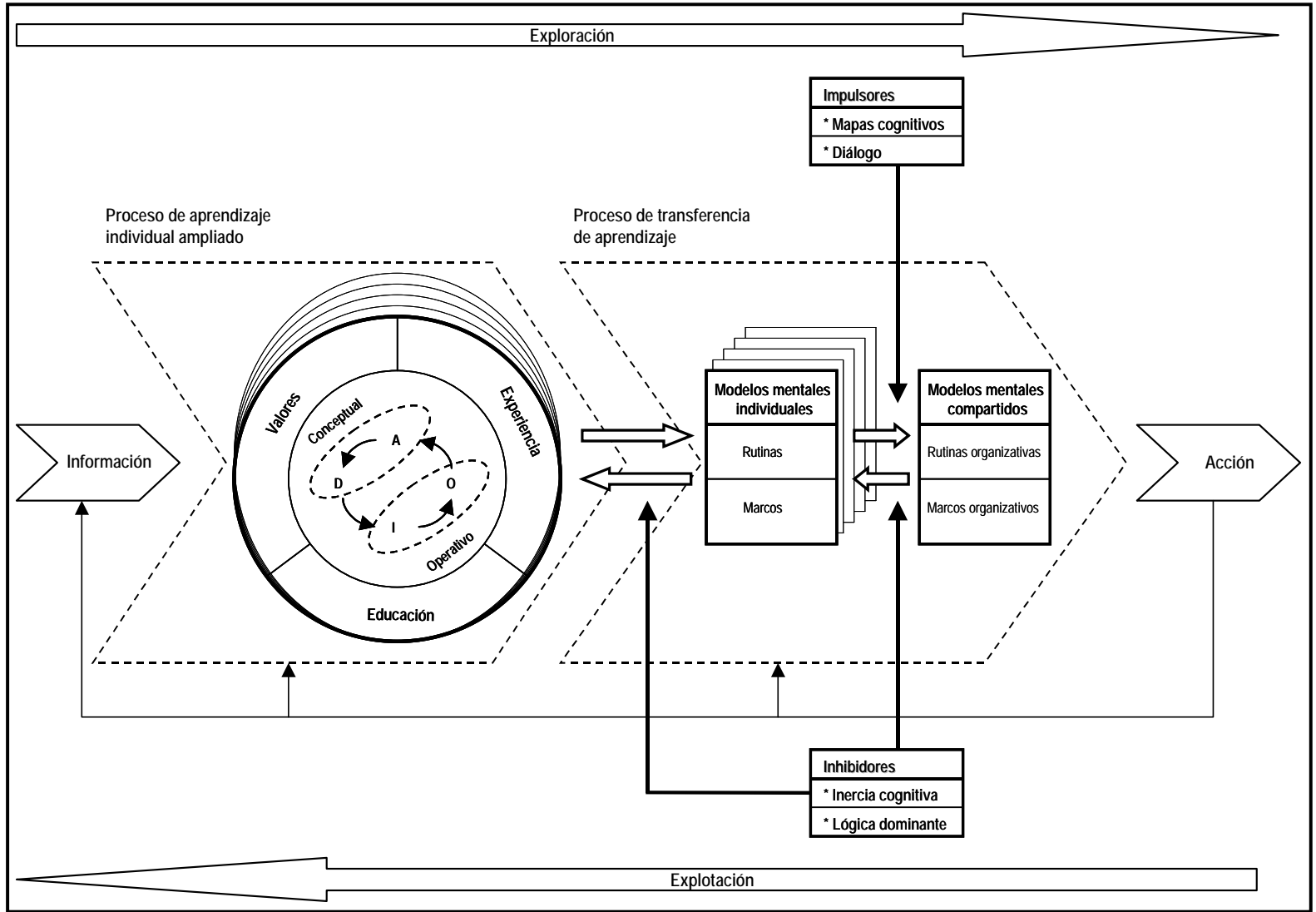




\section{Revista Eletrônica de Ciência Administrativa (RECADM) - ISSN 1677-7387 \\ Faculdade Cenecista de Campo Largo - Coordenação do Curso de Administração v. 3, n. 2, nov./2004 - http://revistas.facecla.com.br/index.php/recadm/}

que se atribuye a los modelos mentales se debe a que buena parte del conocimiento de las organizaciones reside en la mente de los individuos. De esto se deriva, por una parte, que para que se produzca el aprendizaje organizativo es necesario la generación de modelos mentales compartidos que dirijan la acción de la organización y, por otra, que el aprendizaje organizativo será más eficiente cuanto más accesibles sean los modelos mentales de las personas.

Los elementos que se enumeran en la Premisa 4 se analizan en las secciones que vienen más adelante. No obstante, se puede apuntar que los dos primeros -mapas cognitivos y diálogofavorecen la transferencia de aprendizaje, mientras que los dos últimos -inercia cognitiva y lógica dominante- limitan dicha transferencia. La Premisa 5 sostiene que los modelos mentales afectan la acción, y viceversa (Crossan, Lane y White, 1999). De esta forma, los modelos mentales pueden ejercer una influencia sobre la acción: (1) orientándola; (2) mejorándola; y (3) de tipo afectiva (Menon y Varadarajan, 1992). Al mismo tiempo la acción puede ir por delante de los modelos mentales compartidos, esto significa que la organización a través de la experimentación puede iniciar su proceso de aprendizaje (Mintzberg y Westley, 2001).

La Premisa 6 afirma que el aprendizaje organizativo implica la tensión existente entre la exploración y la explotación (Crossan, Lane y White, 1999). Este proceso de interacción mantiene la relación entre los procesos cognitivos y la acción. Así, la exploración suele asociarse a la transferencia de aprendizaje desde el ámbito individual al ámbito colectivo; mientras que la explotación se identifica con la utilización que se hace del aprendizaje que ha quedado institucionalizado, a través de los modelos mentales compartidos, por parte de los sujetos que componen la organización. En definitiva, las empresas tendrán que buscar un equilibrio entre la exploración y la explotación para mantener su competitividad en el largo plazo (March, 1991).

\section{EL APRENDIZAJE EN EL ÁMBITO INDIVIDUAL}

Antes de iniciar el análisis de los procesos de aprendizaje es necesario poner de manifiesto las diferencias existentes entre información y conocimiento. En el caso de la información, uno de sus rasgos distintivos es que ésta no tiene utilidad para el individuo si no es sometida a un proceso de aprendizaje. Asimismo, la información se caracteriza por tener asociado un proceso de selección y preferencia, que será el que determine si dicha información es relevante para el sujeto y, por lo tanto, si es susceptible de ser utilizada en una determinada acción (Selva, 2001).

Para poner de manifiesto este hecho se puede argumentar que, dos personas ante la misma información pueden tener interpretaciones totalmente diferentes, obteniendo como resultado un conocimiento distinto. Esto se debe al conocimiento previo con el que cuenta el sujeto que interioriza dicha información. El conocimiento por consiguiente se distingue de la información, ya que éste es el resultado de un proceso de transformación en la mente del individuo, es decir, de un proceso de aprendizaje $^{4}$. A partir de aquí se desprende que la información existe independientemente del sujeto, mientras que el conocimiento precisa de un "conocedor", convirtiéndolo de este modo en una actividad inherente al ser humano (Arboníes, 2001). 
Revista Eletrônica de Ciência Administrativa (RECADM) - ISSN 1677-7387

Faculdade Cenecista de Campo Largo - Coordenação do Curso de Administração v. 3, n. 2, nov./2004 - http://revistas.facecla.com.br/index.php/recadm/

Teniendo en cuenta las líneas precedentes, parece razonable que si queremos profundizar en el estudio de los procesos de aprendizaje, se debe iniciar la construcción del modelo por el aprendizaje que se produce en el ámbito individual (Simon, 1991). No obstante, antes de comenzar dicho examen es preciso definir "aprendizaje". De acuerdo con Williams (2001, p.68) "el aprendizaje es el proceso en el que se producen cambios relativamente estables en la forma en que vemos las cosas y nos comportamos en la consecución de nuestros objetivos". Esta definición reconoce que el aprendizaje puede ejercer una influencia, tanto en la acción como en los procesos cognitivos, además de que los cambios que tienen lugar son relativamente estables. La ventaja que se deriva de su utilización es que se puede aplicar tanto a los individuos como a las organizaciones, debido a que ambos ven o hacen las cosas en base a las experiencias que han sufrido para la consecución de sus objetivos.

Ahora bien, es conveniente plantearse las siguientes cuestiones:

(1) ¿Qué es el ciclo de aprendizaje individual?

(2) ¿Qué son los modelos mentales?

(3) ¿Qué es el aprendizaje implícito?

Con relación a la primera pregunta, en esta investigación se presenta un ciclo de aprendizaje individual ampliado que se construye a partir del trabajo de Kim (1993). El ciclo de aprendizaje consta de cuatro etapas claramente diferenciadas tales como: (1) observar (O); (2) analizar (A); (3) diseñar (D); y (4) implantar (I). Las personas de acuerdo con este ciclo de aprendizaje experimentarían con determinadas acciones para después observar de forma activa qué es lo que está sucediendo. A continuación analizan tanto consciente como inconscientemente su experiencia, reflexionando sobre sus observaciones, para después diseñar o construir un concepto abstracto que se deriva de dicho análisis. Posteriormente evalúan el concepto abstracto a través de su implantación en un ámbito determinado, que a su vez conduce a una experiencia específica nueva, iniciándose otra vez el ciclo de aprendizaje ${ }^{5}$. Si bien este ciclo de aprendizaje individual recoge desde un punto de vista abstracto las etapas que se incluyen en el proceso de aprendizaje individual, resulta insuficiente puesto que no contempla la influencia

que ejercen en dicho contexto otro tipo de variables ${ }^{6}$ tales como: (1) los valores; (2) la experiencia; y (3) la educación del sujeto.

En lo que se refiere a los modelos mentales, se pueden definir como "un marco interno de referencia, que recrea en la mente del individuo un modelo de la realidad externa" (Picken y Dess, 1998, p.42). La utilización de los modelos mentales, por consiguiente, se debe a la capacidad limitada

\footnotetext{
${ }^{4}$ En el trabajo de Bueno (1998) este análisis se amplia hasta el ámbito de la realidad social. En el estudio se argumenta cuál ha sido el proceso por el que se ha llegado a la Sociedad del Conocimiento. Según este autor se pueden identificar tres fases: (1) proceso de datos; (2) proceso de aprendizaje; y (3) proceso de creación mental.

${ }^{5}$ Kim (1993) parte de la base de que este ciclo consta de dos subprocesos: en primer lugar, la adquisición de habilidades o "know how", que implica la consecución de una habilidad física para realizar un determinado tipo de acción (i.e., aprendizaje operativo). En segundo lugar, la adquisición de "know why", que supone la habilidad de articular un entendimiento conceptual a partir de la experiencia (i.e., aprendizaje conceptual).

6 Entre los trabajos que defienden la importancia de alguna de estas variables en el ciclo de aprendizaje individual se encuentran: Mintzberg (1994); Fondas y Weirsema (1997); y Hamel y Prahalad (1993).
} 


\section{Revista Eletrônica de Ciência Administrativa (RECADM) - ISSN 1677-7387 Faculdade Cenecista de Campo Largo - Coordenação do Curso de Administração v. 3, n. 2, nov./2004 - http://revistas.facecla.com.br/index.php/recadm/}

de la mente humana para procesar información ${ }^{7}$. Esto implica, como sostienen Day y Nedungadi (1994), reconocer lo siguiente: en primer lugar, el entorno no es una realidad inequívoca. La razón es que, constructos como pueden ser las barreras de entrada, fuerzas competitivas, y segmentos de mercado entre otros, adquieren su sentido a través de un proceso interpretativo. En segundo lugar, los individuos deben reducir o absorber en la medida de lo posible la incertidumbre que se genera en el entorno, sirviéndose para este propósito de los modelos mentales. Examinado el concepto de los modelos mentales, cabría preguntarse qué relación existe entre el proceso de aprendizaje individual y estas representaciones de la realidad. Las investigaciones sobre este fenómeno sugieren que, a lo largo del tiempo, a partir del proceso de aprendizaje individual van a generarse una serie de modelos mentales, que son los que finalmente forjen la forma en que se interpreta el mundo que nos rodea ${ }^{8}$ (Daft y Weick, 1984; Kim, 1993; Markides, 1997).

Por último, completando el análisis en el ámbito del aprendizaje individual, se deben explicar los procesos de aprendizaje implícito, es decir, la intuición. Este fenómeno es fundamental para comprender cómo las personas son capaces de entender algo nuevo sin que haya existido una explicación previa. Si bien la intuición ha sido definida de diferentes formas ${ }^{9}$, los elementos comunes a todas estas perspectivas son: (1) la intuición ocurre a un nivel inconsciente; (2) implica una interpretación holística de la información; y (3) lleva aparejada un sentimiento afectivo de que es correcto el pensamiento o la idea surgida (Shapiro y Spence, 1997). En este sentido, entre los estudios más influyentes se encuentra el de Simon (1987) en el que se defiende que la intuición es un proceso de reconocimiento de patrones ${ }^{10}$. De esta forma, la experiencia es la que permite a los individuos organizar la información de tal forma que puedan almacenarla y recuperarla fácilmente.

\section{LA TRANSFERENCIA DE APRENDIZAJE}

¿Cómo se transfiere el aprendizaje que se produce en el ámbito individual al organizativo, y viceversa? Un elemento de gran utilidad para poder explicar la relación que existe entre el aprendizaje individual y el aprendizaje organizativo es la memoria organizativa. No obstante, definir la memoria organizativa como "la información almacenada procedente de la historia de la organización, que puede ser utilizada, para apoyar las decisiones actuales" (Walsh y Ungson, 1991, p.61), sólo llevaría a una indefinición del núcleo básico que genera la dinámica real: la memoria activa, es decir, los modelos mentales individuales y compartidos, ya que como defiende Kim (1993) van a ser los que

\footnotetext{
${ }^{7}$ El uso de los modelos mentales, de acuerdo con Sull (1999), se debe a que es una forma rápida de dar respuesta a una serie de cuestiones clave tales como: (1) ¿En qué negocios estamos?; (2) ¿Cómo se crea valor?; (3) Quiénes son nuestros competidores?; y (4) ¿Cuáles son nuestros consumidores cruciales?

${ }^{8}$ Siguiendo los planteamientos de Kim (1993), tanto el aprendizaje operativo como el aprendizaje conceptual se pueden relacionar con dos partes de los modelos mentales: (1) las rutinas; y (2) los marcos. El aprendizaje operativo es capturado en lo que se conoce como rutinas, que recogen los pasos a llevar a cabo para ejecutar tareas. Asimismo, este aprendizaje operativo es acumulativo y puede a su vez cambiar la rutinas previamente adquiridas, las cuales también pueden condicionar el aprendizaje operativo. El proceso de interacción descrito previamente se produce de forma similar en el caso del aprendizaje conceptual; y dicho aprendizaje cuestiona por qué se llevan a cabo las acciones de una determinada forma. Este tipo de aprendizaje, por su propia naturaleza, puede alterar los marcos en los modelos mentales. Estos nuevos marcos al mismo tiempo pueden aportar una óptica diferente en la resolución de problemas.

${ }_{9}^{9}$ Para profundizar en las distintas interpretaciones sobre la intuición, ver el estudio de Burke y Miller (1999).

${ }^{10}$ En el trabajo de Hayashi (2001) se argumenta que en el ámbito de la gestión en general, las personas que tienen una experiencia más variada y con antecedentes más diversos son más valiosos, puesto que aprenderán de forma más rápida al ser capaces de reconocer más patrones.
} 
Revista Eletrônica de Ciência Administrativa (RECADM) - ISSN 1677-7387

Faculdade Cenecista de Campo Largo - Coordenação do Curso de Administração v. 3, n. 2, nov./2004 - http://revistas.facecla.com.br/index.php/recadm/

definan a qué presta atención una organización, cómo debe actuar, y qué debe recordar de su experiencia.

En esta línea, hacer explícitos los modelos mentales individuales favorecerá el desarrollo de los modelos mentales compartidos, por lo que el aprendizaje organizativo, desde esta perspectiva, es independiente de los sujetos que componen la organización. Además, puesto que gran parte del conocimiento de ésta reside en la mente de los individuos, esto justificaría el énfasis en hacer evidentes los modelos mentales; sobre todo si se tiene en cuenta que dichos modelos son los que permiten que sea accesible la memoria organizativa al resto de la organización ${ }^{11}$. En la Figura 1 se pone de manifiesto que el aprendizaje organizativo no depende de ningún miembro específico de la organización. También se puede observar cómo es factible el aprendizaje individual sin que exista aprendizaje organizativo. Todo esto se muestra de forma gráfica tanto por los diversos círculos que representan el aprendizaje individual, como por los múltiples cuadriláteros que simbolizan los modelos mentales individuales.

Además, cabe apuntar que en el presente modelo conceptual no se recoge el nivel de aprendizaje grupal, al considerarse a los sujetos que componen el grupo como una microorganización (Kim, 1993). Cada una de estas micro-organizaciones tendrá su conjunto de modelos mentales, que van a interactuar con los modelos mentales compartidos, contribuyendo de esta forma en el aprendizaje organizativo; de ahí, que se entienda al grupo como a un "individuo colectivo" (Weick y Roberts, 1993; Hayes y Allinson, 1998). El aprendizaje organizativo en consecuencia dependerá de la capacidad que tengan los individuos para hacer más eficientes sus modelos mentales.

La determinación del mecanismo de transferencia entre el aprendizaje individual y organizativo, hace que adquiera especial relevancia el análisis de los siguientes elementos, que guardan relación con dicho proceso:

(1) Impulsores: mapas cognitivos y diálogo.

(2) Inhibidores: inercia cognitiva y lógica dominante.

Los mapas cognitivos, según Langfield-Smith (1992, p.250), se pueden definir como "una descripción gráfica de las distintas formas en que los individuos ven un dominio concreto -campo de pensamiento o acción-". Los mapas cognitivos, por lo tanto, son capaces de representar las abstracciones que un individuo tiene de la realidad: sus modelos mentales. Hacer accesibles estos modelos facilita la transferencia de aprendizaje desde el ámbito individual al organizativo, lo que les hace ser una poderosa herramienta en el aprendizaje organizativo, ya que permite "operativizar" el concepto de modelo mental ${ }^{12}$. Esto convierte a los mapas cognitivos en una forma de representación que favorece

\footnotetext{
${ }^{11}$ Dixon (1997) distingue los siguientes motivos por los que los modelos mentales no son siempre accesibles al resto de la organización: (1) personales; y (2) políticos o logísticos.

12 El uso de mapas cognitivos para examinar los modelos mentales tiene importantes repercusiones para el aprendizaje, como son: (1) ayuda a entender cómo se abordan cada una de las etapas del proceso de aprendizaje; (2) permite valorar la calidad de las ideas generadas; (3) facilita la determinación de si el sujeto posee una perspectiva limitada sobre un determinado fenómeno; y (4) ayuda a entender cuál es el proceso de selección y preferencia de información que siguen los modelos mentales.
} 
Revista Eletrônica de Ciência Administrativa (RECADM) - ISSN 1677-7387

Faculdade Cenecista de Campo Largo - Coordenação do Curso de Administração v. 3, n. 2, nov./2004 - http://revistas.facecla.com.br/index.php/recadm/

el análisis y la comparación de modelos mentales (Spicer, 1998; Jenkins y Johnson, 1997; Clarke y Mackanes, 2001).

Lo indicado implica la posibilidad, por parte del sujeto, de participar de forma activa en la exploración de sus modelos mentales. Dichos planteamientos son bastante reveladores, ya que al perfeccionar los modelos mentales individuales se está facilitando el aprendizaje organizativo, siempre y cuando estos modelos mentales sean de utilidad para la organización y hayan sido explicitados. No obstante, los mapas cognitivos representan de forma gráfica solamente un modelo mental para un ámbito concreto de actuación (Swan, 1995). Este mapa cognitivo (i.e., modelo mental revelado) sería asimismo una representación aproximada del modelo mental, debido a que en este tipo de representaciones es imposible recoger el conjunto total de relaciones, al ser muy complejo y poco práctico ${ }^{13}$.

En el caso del diálogo, si bien la representación gráfica de los modelos mentales -mapas cognitivos- es importante para desarrollar un entendimiento compartido, también el diálogo contribuye en este proceso. El diálogo, de acuerdo con Isaacs $\left(1993\right.$, p.26) ${ }^{14}$, es "una disciplina de pensamiento colectivo e indagación; un proceso en el que se transforma la calidad de la conversación y, en concreto, el pensamiento que reside bajo ésta". La definición parte de la premisa de que los seres humanos actúan frecuentemente dentro de una serie de supuestos compartidos -modelos mentales compartidos- que van a ir evolucionando a lo largo del tiempo. El diálogo por consiguiente busca que las personas aprendan a pensar de manera conjunta, no sólo en un sentido de análisis y resolución de problemas, sino también en el de comprender las reglas de tipo cognoscitivo que dirigen su pensamiento y acción ${ }^{15}$.

De acuerdo a lo anterior, se puede afirmar que el diálogo tiene un papel destacado en el proceso de representación gráfica de los modelos mentales -mapas cognitivos-, puesto que posibilita expresar aquello que había sido para los individuos: presentimientos, emociones, o sensaciones; lo que convierte al diálogo en un impulsor del aprendizaje organizativo. En consecuencia, el diálogo pretende descubrir e indagar la relación que existe entre las estructuras interpretativas internas de pensamiento y cómo éstas nos hacen comprender la realidad que nos rodea. De esta forma, revelar los modelos mentales es fundamental para el aprendizaje organizativo; sobre todo, porque estas interpretaciones son las que producen una respuesta física (i.e., acción).

En lo que se refiere a la inercia cognitiva, Hodgkinson (1997, p.922) la define como "la inhabilidad para revisar los modelos mentales con la suficiente rapidez como para incorporar las condiciones cambiantes del entorno". La inercia cognitiva tal y como se plantea tendrá mayores implicaciones cuando las empresas se encuentren en entornos dinámicos, ya que los procesos de aprendizaje irán por detrás de las alteraciones que se producen en éste. En otras palabras, como los modelos mentales se generan a partir del proceso de aprendizaje individual, y estos modelos a su vez

\footnotetext{
${ }^{13}$ Los mapas cognitivos ya sean individuales o compartidos, no son más que interpretaciones sobre un determinado ámbito (e.g., estructura de mercado, competidores, tipo de consumidores) con lo que son altamente subjetivos e idiosincrásicos (Barr y Huff, 1997)

${ }^{14}$ En 1993, William N. Isaacs era director del "Proyecto Diálogo" en el Centro de Aprendizaje Organizativo del M.I.T., perteneciente al Sloan School of Management.
} 
Revista Eletrônica de Ciência Administrativa (RECADM) - ISSN 1677-7387

Faculdade Cenecista de Campo Largo - Coordenação do Curso de Administração v. 3, n. 2, nov./2004 - http://revistas.facecla.com.br/index.php/recadm/

interaccionan con el propio proceso de aprendizaje, si los modelos mentales no evolucionan para ajustarse a la realidad, el proceso de aprendizaje tampoco lo hará. Desde esta perspectiva, se deduce que la capacidad para competir de la empresa quedaría limitada por su inhabilidad para responder de forma efectiva al entorno.

Asimismo, la importancia de este inhibidor reside en que, como los modelos mentales están asociados a los procesos de atención e interpretación de la información (i.e., estímulos), con el paso del tiempo estos modelos mentales tienen tendencia a centrarse siempre en los mismos puntos de referencia, derivándose de dicho proceso la incapacidad para detectar nuevas oportunidades. Por lo tanto, la información que no sea habitual no capturará la atención de la empresa ${ }^{16}$.

Por otra parte, las preguntas a resolver a continuación serían: (1) ¿Qué es la lógica dominante?; (2) ¿Cuál es la diferencia primordial entre la inercia cognitiva y la lógica dominante?; y (3) ¿Cómo se relacionan ambos conceptos? Prahalad y Bettis (1986, p.490) se refieren a la lógica dominante como "la forma en que los directivos conceptualizan la empresa y toman decisiones críticas de asignación de recursos - ya sea en tecnologías, desarrollo de productos, distribución, publicidad, o en recursos humanos .... siendo almacenada -lógica dominante- a través de modelos mentales". De la definición se puede derivar que la lógica dominante es, en definitiva, un conjunto de modelos mentales.

Con relación a la segunda pregunta, se puede indicar que la inercia cognitiva consiste en la inhabilidad para revisar los modelos mentales a medida que cambia el entorno; mientras que la lógica dominante se centra en el grado de influencia que los modelos mentales que la integran, a través del proceso de refuerzo e interacción social ${ }^{17}$, pueden ejercer sobre el resto de modelos mentales de la organización. Finalmente, se puede afirmar que si partimos de la base de que la lógica dominante es un cúmulo de modelos mentales que pueden ejercer su influencia, tanto en el ámbito individual como colectivo, este concepto se puede relacionar con la inercia cognitiva en el sentido de que, si la lógica dominante no se revisa para albergar los cambios del entorno, se estaría produciendo un fenómeno de inercia cognitiva ${ }^{18}$.

Finalmente, una de las premisas del modelo conceptual es que los modelos mentales compartidos ejercen una influencia sobre la acción, y viceversa. Las acciones organizativas en esta línea serán de utilidad puesto que aportarán nuevos datos para que sean interpretados, tanto en el ámbito individual como colectivo. Esto implica que las cuatro etapas (i.e., información, proceso de aprendizaje individual ampliado, proceso de transferencia de aprendizaje, y acción) estén interconectadas a través de un bucle de retroalimentación como se muestra en la Figura 1. En

\footnotetext{
${ }^{15}$ Para profundizar sobre esta cuestión véase el trabajo de Argyris $(1991,1994)$ en el que se muestra la influencia que pueden ejercer las "teorías de la acción" en el diálogo.

${ }^{16} \mathrm{Si}$ los modelos mentales no evolucionan, la perspectiva de análisis tampoco lo hace. De esto se desprende la dificultad para descubrir nuevas oportunidades de mercado. Existen algunos estudios que han tratado de profundizar sobre este hecho, entre los que se pueden destacar: Miller (1994); Miller y Chen (1994); Drucker (1994); Tushman y O’Reilly (1996); y Barr y Huff (1997).

17 Picken y Dess (1998) argumentan que, aunque los individuos interpretan la realidad de forma diferente, el proceso de socialización y acomodación dentro de una empresa, suele producir a lo largo del tiempo un conjunto amplio de supuestos compartidos acerca de la organización y la industria.

${ }^{18}$ Algunos autores como Bettis y Prahalad (1995) argumentan que la lógica dominante actúa como un filtro, que incide en los estímulos que se perciben.
} 
Revista Eletrônica de Ciência Administrativa (RECADM) - ISSN 1677-7387

Faculdade Cenecista de Campo Largo - Coordenação do Curso de Administração v. 3, n. 2, nov./2004 - http://revistas.facecla.com.br/index.php/recadm/

consecuencia, los modelos mentales compartidos que se generan del proceso de transferencia de aprendizaje, van a condicionar las acciones de la organización, y éstas al mismo tiempo, dichos modelos mentales compartidos.

\section{COMPRENSIÓN DE LOS PROCESOS DE APRENDIZAJE ORGANIZATIVO. LA HISTORIA DE MIGHTY MOTORS INC ${ }^{19}$.}

Con el objetivo de mejorar el entendimiento de los procesos de aprendizaje organizativo se utilizará la "historia de aprendizaje" ${ }^{20}$ de Mighty Motors Inc. A principios de la década de los noventa, la mayoría de los altos directivos de esta empresa estaban preocupados por la inhabilidad para seguir innovando en el desarrollo de productos. La innovación en este sentido se consideraba la piedra angular para la supervivencia a largo plazo de la empresa. El problema es que no eran capaces de mejorar el proceso de desarrollo de productos. Para hacer frente a esta situación se optó por seguir un enfoque basado en el aprendizaje. Los resultados de esta iniciativa de cambio fueron:

\footnotetext{
Mighty Motors tiene motores que sus clientes aman y ha incrementado la capacidad de producción hasta duplicar los niveles de hace una década. Con una expansión en su línea de productos, están alcanzando nuevos mercados. Además, la demanda de nuevos productos por parte del cliente, implica un crecimiento y expansión continuos (Castleberg y Roth, 1998, p.71).
}

Se pueden distinguir tres grandes fases en esta iniciativa de cambio: en la primera, una serie de directivos identifican la necesidad de cambiar el modelo mental compartido ${ }^{21}$ que dirige la acción en el área de desarrollo de productos. Esto es posible como resultado de la interpretación que hacen, a través de sus modelos mentales individuales, de la información que reciben tanto del medio interno como externo de la organización; siendo también necesario que no se haya producido un fenómeno de inercia cognitiva y/o lógica dominante:

\footnotetext{
La mayoría de los directivos de Mighty Motors, había expresado su preocupación, de una forma u otra, sobre la habilidad de la compañía para seguir innovando.... pero una escasez de producto llevó a largas discusiones sobre los problemas a los que se enfrentaba en el desarrollo de productos.... Cuando el grupo examinó el proceso de desarrollo de productos, descubrió una serie de hechos fatales que solían impedir el progreso.... Una vez reconocidos los problemas adquirió sentido centrar los esfuerzos de aprendizaje en este área (Castleberg y Roth, 1998, pp.2-4).
}

En la segunda fase, se crea un equipo de aprendizaje en el área de desarrollo de productos y se utilizan herramientas de aprendizaje. Los directivos en este sentido construyen un mapa cognitivo con el que adquieren conciencia de los principales problemas del sistema de desarrollo de productos. Durante su elaboración el diálogo tiene un papel determinante, puesto que examina las estructuras

\footnotetext{
${ }^{19}$ Si bien el estudio ha sido realizado por Castleberg y Roth (1998), en la presente sección se ha seguido el enfoque metodológico de Sugarman (2001), es decir, a partir de un nuevo análisis de la "historia de aprendizaje", se persigue ilustrar el modelo conceptual que se propone en los epígrafes precedentes. Además, la utilización de la "historia de aprendizaje" para explicar el modelo conceptual, como sostienen Crossan, Lane y White (1999), no significa que sea un soporte empírico del mismo. Por otra parte, con relación al nombre de Mighty Motors Inc., hay que señalar que se trata de un seudónimo, aunque también hay que poner de manifiesto que es una empresa americana, que se dedica a la fabricación de motores, sin ser una de las tres grandes del sector (i.e., General Motors -GM-, Ford, y Chrysler).

${ }^{20}$ Para profundizar sobre la metodología que se emplea en la elaboración de una "historia de aprendizaje" véase: Kleiner y Roth (1997); y Roth y Kleiner (1998).

${ }^{21}$ Siguiendo a Crossan, Lane y White (1999), las palabras en letra cursiva hacen referencia a conceptos que forman parte del modelo conceptual propuesto en la presente investigación.
} 


\section{Revista Eletrônica de Ciência Administrativa (RECADM) - ISSN 1677-7387 Faculdade Cenecista de Campo Largo - Coordenação do Curso de Administração v. 3, n. 2, nov./2004 - http://revistas.facecla.com.br/index.php/recadm/}

internas de pensamiento y cómo éstas producen una respuesta física en un determinado ámbito de actuación:

\footnotetext{
Los mapas cognitivos dieron a Mighty Motors una nueva forma de ver el proceso de desarrollo de productos..... Los ejecutivos comenzaron a comprender y comunicarse con otros la naturaleza sistémica de lo que había sido considerado previamente un problema del área de desarrollo de productos.... La creación del mapa cognitivo representó el primer esfuerzo de Mighty Motors en integrar y aplicar las habilidades de aprendizaje en una situación real.... De esta modo, la gente era capaz de entender los problemas básicos en el proceso de desarrollo de productos de forma más clara (Castleberg y Roth, 1998, pp.5-6).
}

En la última fase, se contrata a un nuevo director de ingeniería con la tarea de "reorganizar" el área de desarrollo de productos. En definitiva, se incorpora una persona con unos valores, experiencia y educación específicos, que den garantías para llevar a cabo un proceso de aprendizaje organizativo de estas características. La medida se ve complementada con la construcción de un centro de desarrollo de productos, en el que se incorpora la "nueva filosofía" sobre cómo debían ser diseñados y entregados los productos:

En la primavera de 1993, se contrata un nuevo director ingeniería y se le pone a cargo de la "reinvenvión" de desarrollo de productos.... Habiendo trabajado para una corporación mayor, el nuevo director de ingeniería trajo consigo mismo considerable conocimiento y experiencia en el desarrollo de producto. Él entendía los detalles sobre cómo gestionar legiones de ingenieros en una gran organización y cómo diseñar el proceso requerido para conectar múltiples funciones.... El nuevo director de ingeniería y el vicepresidente de ingeniería vieron en un nuevo edificio la forma para hacer los cambios necesarios en el proceso de desarrollo de productos.... Este nuevo centro apoyaría el cambio de filosofía.... (Castleberg y Roth, 1998, pp. 25-29).

\section{CONCLUSIONES}

En un artículo en el que se analizaba cómo se transfiere el aprendizaje individual hacia el resto de la organización, Kim (1993, p.49) pone de manifiesto la necesidad de seguir profundizando en el estudio del papel desempeñado por los modelos mentales en dicho contexto:

Más trabajo se necesita para una mejor comprensión del papel de los modelos mentales en el aprendizaje individual y organizativo, los tipos de modelos mentales que son apropiados para representar la complejidad dinámica, ....y los medios a través de los cuales nuevo aprendizaje puede ser transferido al resto de la organización.

Por otra parte, en una investigación en la que se examinaba el fenómeno del aprendizaje organizativo, desde un punto de vista integrador y reconociendo la importancia de los procesos sociales y psicológicos, Crossan, Lane y White (1999, p.535) resaltan también la necesidad de comprender qué elementos pueden favorecer o limitar la transferencia de aprendizaje desde el ámbito individual al organizativo, y viceversa:

Mientras que este marco debería de fomentar y ayudar en la búsqueda de una comprensión más holística del aprendizaje organizativo, hay.... áreas de investigación en particular que ayudarán a avanzar la teoría. La primera es comprender los mecanismos que mejoran o restringen los "stocks" y flujos de aprendizaje. 
Revista Eletrônica de Ciência Administrativa (RECADM) - ISSN 1677-7387

Faculdade Cenecista de Campo Largo - Coordenação do Curso de Administração v. 3, n. 2, nov./2004 - http://revistas.facecla.com.br/index.php/recadm/

El modelo conceptual de aprendizaje organizativo que aquí se propone trata de hacer frente en parte a las necesidades de investigación planteadas por los autores precedentes. No obstante, las dificultades para tratar de alcanzar este objetivo han sido de dos tipos: en primer lugar, la incorporación de una multitud de trabajos debido a la naturaleza multi-disciplinar del fenómeno analizado, que ha supuesto una compleja labor de síntesis. En segundo lugar, la escasa literatura especializada que examina la labor desempeñada por los modelos mentales en este campo de estudio. Asimismo, en cuanto a las futuras líneas de investigación, de gran interés científico, que podrían complementar el modelo planteado están: por una parte, incorporar la escuela de poder en el análisis del aprendizaje organizativo; por otra, examinar cómo las interacciones entre las distintas subculturas que residen en una organización pueden condicionar el aprendizaje organizativo. Por último, cabe señalar que, con relación al modelo presentado, el siguiente paso sería llevar a cabo una validación empírica del mismo.

\section{REFERENCIAS}

Arboníes, A. L. (2001), "Cómo evitar la miopía en la gestión del conocimiento", Díaz de Santos, Madrid.

Argyris, C. (1977), "Double loop learning in organizations", Harvard Business Review, September-October, pp.115-125.

Argyris, C. (1991), "Teaching smart people how to learn", Harvard Business Review, May-June, pp.99-109.

Argyris, C. (1994), "Good communication that blocks learning", Harvard Business Review, July-August, pp.77-85.

Barr, P. S. y Huff, A. S. (1997), "Seeing isn't believing: understanding diversity in timing strategic response", Journal of Management Studies, Vol. 34 no. 3, pp.337-370.

Bettis, R. A. y Prahalad, C. K. (1995), "The dominant logic: restrospective and extension", Strategic Management Journal, Vol. 16, pp.5-14.

Bueno, E. (1998), "El capital intangible como clave estratégica en la competencia actual", Boletín de Estudios Económicos, Vol. LIII nº. 164, pp.207-229.

Bueno, E. (2001), "Propuesta integradora del concepto de dirección del conocimiento", Capítulo 14, pp.253-268. En: Arboníes, L. A. (2001), "Cómo evitar la miopía en la gestión del conocimiento", Díaz de Santos, Madrid.

Burke, L. A. y Miller, M. K. (1999), "Taking the mystery out of intuitive decision making", Academy of Management Executive, Vol. 13 no 4, pp. 91-99.

Cangelosi, V. E. y Dill, W. R. (1965), "Organizational learning: observations toward a theory", Administrative Science Quarterly, Vol. 10, pp.175-203.

Castleberg, M. y Roth, G. (1998), "The learning initiative at Mighty Motors Inc.", The Society for Organizational Learning (SoL), Cambridge, Massachussets.

Clarke, I. y Mackaness, W. (2001), "Management intuition: an interpretative account of structure and content of decision schemas using cognitive maps", Journal of Management Studies, Vol. 38 n² 2, pp.147-172.

Crossan, M. M.; Lane, H. W. y White, R. E. (1999), "An organizational learning framework: from intuition to institution", Academy of Management Review, Vol. 24 no 3, pp.522-537.

Daft, R. L. y Weick, K. E. (1984), "Toward a model of organizations as interpretation systems", Academy of Management Review, Vol. 9 nº 2, pp.284-295.

Day, G. S. y Nedungadi, P. (1994), "Managerial representations of competitive advantage", Journal of Marketing, April, pp.31-44. 
Revista Eletrônica de Ciência Administrativa (RECADM) - ISSN 1677-7387

Faculdade Cenecista de Campo Largo - Coordenação do Curso de Administração

v. 3, n. 2, nov./2004 - http://revistas.facecla.com.br/index.php/recadm/

Dixon, N. M. (1997), "The hallways of learning”, Organizational Dynamics, Vol. 25 n 4, pp.23-34.

Drucker, P. F. (1992), "The new society of organizations", Harvard Business Review, September-October, pp.95104.

Drucker, P. F. (1994), "The theory of the business", Harvard Business Review, September-October, pp.95-104.

Fiol, C. M. y Lyles, M. A. (1985), "Organizational learning", Academy of Management Review, Vol. 10 n4, pp.803-813.

Fondas, N. y Weirsema, M. (1997), "Changing of the guard: the influence of CEO socialization on strategic change", Journal of Management Studies, Vol. 3 nº 4, pp. 561-584.

Garvin, D. (1993), "Building a learning organization", Harvard Business Review, July-August, pp.78-91.

Hamel, G. y Prahalad, C. K. (1993), "Strategy as stretch and leverage", Harvard Business Review, March-April, pp.75-84.

Harvey, C. y Denton, J. (1999): "To come of age: the antecedents of organizational learning", Journal of Management Studies, Vol. 36 No. 7, pp.897-918.

Hayashi, A. M. (2001), "When to trust your gut", Harvard Business Review, February, pp.59-65.

Hayes, J. y Allinson, C. W. (1998), "Cognitive style and the theory and practice of individual and collective learning in organizations", Human Relations, Vol. 51 no 7, pp.847-871.

Hodgkindon, G. P. (1997), "Cognitive inertia in a turbulent market: the case of UK residential estate agents", Journal of Management Studies, Vol. 34 n 5, pp.921-945.

Huber, G. (1991), "Organizational learning: the contributing processes and the literatures", Organization Science, Vol. 2 n¹, pp.88-115.

Hunt, S. D. y Morgan, R. M. (1995), "The comparative advantage theory of competition", Journal of Marketing, Vol. 59 (April), pp.1-15

Hunt, S. D. y Morgan, R. M. (1996), "The resource advantage theory of competition: dynamics, path dependencies, and evolutionary dimensions", Journal of Marketing, Vol. 60 (October), pp.107-114.

Hunt, S. D. y Morgan, R. M. (1997), "Resource advantage theory: a snake swallowing its tail or a general theory of competition", Journal of Marketing, Vol. 61 (October), pp.74-82.

Hunt, S. D. (2000), "A general theory of competition: Resources, competences, productivity and economic growth", Sage Publications, Thousand Oaks, California.

Instituto Universitario Euroforum Escorial (1998): "Medición del capital intelectual. Modelo Intelect", Madrid, Diciembre.

Isaacs, W. N. (1993), "Taking flight: dialogue, collective thinking, and organizational learning", Organizational Dynamics, Vol. 22 n², pp. 24-40.

Jenkins, M. y Johnson, G. (1997), "Entrepreneurial intentions and outcomes: a comparative causal mapping study", Journal of Management Studies, Vol. 34 n 6, pp.895-920.

Kim, D. H. (1993), "The link between individual and organizational learning", Sloan Management Review, Fall, pp.37-50.

Kleiner, A. y Roth, G. (1997), "How to make experience your company's best teacher", Harvard Business Review, September-October, pp.172-177.

Langfield-Smith, K. (1992), "Exploring the need for a shared cognitive map", Journal of Management Studies, Vol. $29 n^{\circ} 3$, pp.349-369.

Levitt, B. y March, J. G. (1988), "Organizational learning", Annual Review of Sociology, Vol. 14, pp.319-340. 
Revista Eletrônica de Ciência Administrativa (RECADM) - ISSN 1677-7387

Faculdade Cenecista de Campo Largo - Coordenação do Curso de Administração v. 3, n. 2, nov./2004 - http://revistas.facecla.com.br/index.php/recadm/

López, P. E. y López, J. A., (2001a), "Aprendizaje organizativo, dinámica competitiva y procesos de dependencia", XV Congreso Nacional y XI Congreso Hispano Francés AEDEM, Junio, Las Palmas de Gran Canaria.

López, P. E. y López, J. A., (2001b), "El aprendizaje organizativo como un recurso. Un enfoque dinámico", XI Congreso Nacional de ACEDE, Septiembre, Zaragoza.

March, J. G. (1991), "Exploration and exploitation in organizational learning", Organization Science, Vol. 2 n 1 , pp.71-87.

Markides, C. (1997), "Strategic innovation”, Sloan Management Review, Spring, pp.9-23.

Menon, A. y Varadarajan, P. R. (1992), "A model of knowledge use within firms", Journal of Marketing, Vol. $56 \mathrm{n}^{\circ}$ 4, pp.53-71.

Miller, D. (1994), "What happens after success: the perils of excellence", Journal of Management Studies, Vol. 31 n० 3, pp.325-358.

Miller, D. y Chen, M. (1994), "Sources and consequences of competitive inertia: A study of the U.S. airline industry", Administrative Science Quarterly, Vol. 39, pp.1-23.

Mintzberg, H. (1994), "Rounding out the manager's job”, Sloan Management Review, Fall, pp.11-26.

Mintzberg, H. y Westley, F. (2001), "Decision making: it's not what you think", Sloan Management Review, Spring, pp.89-93.

Nonaka, I. (1994), "A dynamic theory of organizational knowledge creation”, Organization Science, Vol. 5 n 1 , pp.14-37.

Picken, J. C. y Dess, G. G. (1998), "Right strategy, wrong problem”, Organizational Dynamics, Summer, pp.3549.

Pondy, L. y Mitroff, I. I. (1979), "Beyond open systems models of organizations", pp. 3-39. En: Staw, M. (Ed.) (1979), "Research in organizational behavior", JAI Press, Greenwich, Conn.

Prahalad, C. K. y Bettis, R. A. (1986), "The dominant logic: a new linkage between diversity and performance", Strategic Management Journal, Vol. 7, pp.485-501.

Priem, R. L. y Butler, J. E. (2001), "Is the resource based view a useful perspective for strategic management research?", Academy of Management Review, Vol. 26 nº 1, pp.22-40.

Roth, G. y Kleiner, A. (1998), "Developing organizational memory through learning histories", Organizational Dynamics, Autumn, pp.43-60.

Selva, M. J. (2001), "Importancia de la gestión del conocimiento. Homo sapiens versus maquina sapiens", $2^{a}$ Conferencia de la Asociación Portuguesa de Sistemas de Información (APSI), Noviembre, Évora (Portugal).

Senge, P. (1990), "The leader's new work: building learning organizations", Sloan Management Review, Fall, pp.7-23.

Senge, P. (1992), "Mental models", Planning Review, March-April, pp.4-10.

Senge, P. (1998), "La quinta disciplina", Granica, Barcelona, Sexta Edición.

Shapiro, S. y Spence, M. (1997), "Managerial intuition: A conceptual and operational framework", Business Horizons, Vol. $40 \mathrm{n}^{\circ} 1$, pp.63-70.

Shrivastava, P. (1983), "A tipology of organizational learning systems", Journal of Management Studies, Vol. 20 no 1, pp.7-28.

Simon, H. (1987), "Making management decisions: the role of intuition and emotion", Academy of Management Executive, February, p.57-64. 
Revista Eletrônica de Ciência Administrativa (RECADM) - ISSN 1677-7387

Faculdade Cenecista de Campo Largo - Coordenação do Curso de Administração

v. 3, n. 2, nov./2004 - http://revistas.facecla.com.br/index.php/recadm/

Simon, H. (1991), "Bounded rationality and organizational learning", Organization Science, Vol. 2 n 1, pp.125134.

Spicer, D. P. (1998), "Linking mental models and cognitive maps as an aid to organizational learning", Career Development International, Vol. 3 n³, pp.125-132.

Stata, R. (1989), "Organizational learning: the key to management innovation", Sloan Management Review, Spring, pp.63-73.

Sugarman, B. (2001), "A learning based approach to organizational change", Organizational Dynamics, Summer, pp.62-76.

Sull, D. N. (1999), “Why good companies go bad?”, Harvard Business Review, July-August, pp.42-52.

Swan, J. A. (1995), "Exploring knowledge and cognitions in decisions about technological innovation: mapping managerial cognitions", Human Relations, Vol. 48 n 11, pp.1241-1270.

Tushman, M. L. y O’Reilly, C. A. (1996), “Ambidextrous organization: managing evolutionary and revolutionary change", California Management Review, Vol. 38 n 4, pp.8-30.

Walsh, J. P. y Ungson, G. R. (1991), "Organizational memory", Academy of Management Review, Vol. $16 \mathrm{n}^{\circ} 1$, pp.57-91.

Weick, K. E. y Roberts, K. H. (1993), "Collective mind in organizations: heedful interrelating on flight decks", Administrative Science Quarterly, Vol. 38, pp.357-381.

Williams, A. O. (2001), "A belief focused process model of organizational learning", Journal of Management Studies, Vol. 38 no 1, pp.67-85. 